\title{
Saúde, espiritualidade e religiosidade na visão dos estudantes de medicina
}

\author{
Health, spirituality and religiosity in the view of medical students
}

Salud, espiritualidad y religiosidad en la mirada de estudiantes de medicina

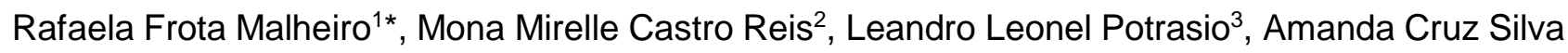
Oliveira ${ }^{2}$, Rebeca Vieira da Silva ${ }^{4}$, Lucas Santana Passinho ${ }^{4}$, Filipe Ribeiro Martins ${ }^{5}$, Lucas Santos Amaral $^{5}$, Jessica Santos de Oliveira ${ }^{6}$, Michela Macedo Lima Costa ${ }^{1}$.

\section{RESUMO}

Objetivo: Compreender as concepções dos estudantes de medicina sobre a influência da espiritualidade e religiosidade na prática clínica e o impacto destas na construção do cuidado e da relação médico-paciente. Revisão bibliográfica: Essa deficiência na abordagem da religiosidade e espiritualidade durante a graduação vai ao oposto do panorama social e cultural do Brasil, uma vez que tais aspectos são muito debatidos e vividos pela população. Outra barreira é o receio de influenciar nas crenças do paciente e ser interpretado negativamente ao abordar tais temáticas na consulta. É importante destacar que abordar espiritualidade e religiosidade é tornar a prática médica mais humanizada, uma vez que mesmo quando a ciência e os tratamentos não conseguem resolver o problema do paciente, o simples fato de ouvir, dar apoio e garantir que essa pessoa vivencie sua espiritualidade pode lhe proporcionar conforto e consolo e assim contribuir para a construção de uma medicina baseada no cuidado. Considerações finais: A religiosidade e espiritualidade, quando bem interpretadas e utilizadas, podem fornecer informações essenciais para compreensão de questões atuais do paciente e ainda impactar positivamente em todas as etapas do processo de cuidado.

Palavras-chave: Espiritualidade, Religião, Saúde.

\begin{abstract}
Objective: To understand the conceptions of medical students about the influence of spirituality and religiosity in clinical practice and their impact on the construction of care and the doctor-patient relationship. Review bibliography: This deficiency in the approach to religiosity and spirituality during graduation goes to the opposite of the social and cultural panorama of Brazil, since such aspects are much debated and experienced by the population. Another barrier is the fear of influencing the patient's beliefs and being interpreted negatively when addressing such issues in the consultation. It is important to highlight that approaching spirituality and religiosity is to make medical practice more humanized, since even when science and treatments cannot solve the patient's problem, the simple fact of listening, giving support and ensuring that this person experiences their spirituality it can provide you with comfort and consolation and thus contribute to the construction of a medicine based on care. Final considerations: Religiosity and spirituality, when well interpreted and used, can provide essential information for understanding the patient's current issues and still positively impact all stages of the care process.
\end{abstract}

Keywords: Spirituality, Religion, Health.

\footnotetext{
${ }^{1}$ Faculdade Santo Agostinho (FASA), Vitória da Conquista - BA. *E-mail: rafaelamalheiro14@hotmail.com

2 Faculdade AGES de Medicina, Jacobina - BA.

${ }^{3}$ Centro Universitário FAMINAS, Muriaé - MG.

${ }^{4}$ Universidade do Estado da Bahia (UNEB), Salvador - BA.

${ }^{5}$ Universidade Estadual de Montes Claros (UNIMONTES), Montes Claros - MG.

${ }^{6}$ Universidade Federal da Bahia (UFBA), Vitória da Conquista - BA.
} 


\section{RESUMEN}

Objetivo: Comprender las concepciones de los estudiantes de medicina sobre la influencia de la espiritualidad y la religiosidad en la práctica clínica y su impacto en la construcción del cuidado y la relación médico-paciente. Revisión bibliográfica: Esta deficiencia en el abordaje de la religiosidad y espiritualidad durante la graduación va al revés del panorama social y cultural de Brasil, ya que tales aspectos son muy discutidos y vividos por la población. Otra barrera es el miedo a influir en las creencias del paciente y ser interpretado negativamente al abordar estos temas en la consulta. Es importante resaltar que acercarse a la espiritualidad y la religiosidad es humanizar la práctica médica, ya que aun cuando la ciencia y los tratamientos no puedan resolver el problema del paciente, el simple hecho de escuchar, dar apoyo y hacer que esta persona experimente su espiritualidad puede brindarle con comodidad y consuelo y así contribuir a la construcción de una medicina basada en el cuidado. Consideraciones finales: La religiosidad y la espiritualidad, cuando son bien interpretadas y utilizadas, pueden proporcionar información esencial para comprender los problemas actuales del paciente y, aún así, impactar positivamente en todas las etapas del proceso de atención.

Palabras clave: Espiritualidad, Religión, Salud.

\section{INTRODUÇÃO}

Desde os seus primórdios, a sociedade encontra a dimensão religiosa e espiritual caminhando lado a lado com o ser humano. Isso porque esses fatores modulam as atitudes das pessoas perante determinadas situações de estresse, sofrimento e principalmente problemas relacionados à saúde, podendo proporcionar uma maior aceitação, paz, autoconfiança e facilitando assim o processo terapêutico (NANTES AC e GRUBITS S, 2017).

A religiosidade e espiritualidade tem demonstrado um impacto potencial sobre a saúde física e mental do ser humano, a partir delas é possível considerar o paciente sobre um ponto de vista global, integrando sua individualidade e sua relação com o meio, permitindo assim a condução de novos horizontes preventivos, diagnósticos e terapêuticos. Com isso, essa relação ganhou muito destaque, uma vez que o ser humano passou a ser considerado "bio-psico-sócio-espiritual", tendo o bem-estar religioso e espiritual como um meio de apoiar e fornecer base para o processo de saúde e doença das pessoas (NANTES AC e GRUBITS S, 2017, DOMINGUES MES, et al., 2020).

Diante disso, tais dimensões são compreendidas como algo inerente ao ser humano, sendo necessário a adoção de um modelo de abordagem médica mais integralista que incorpora o aspecto espiritual do cuidado, compreendendo as necessidades dos pacientes e suas famílias, entendendo o adoecimento pela complexa interação de fatores biológicos, sociais, psicológicos e espirituais. Com tal abordagem, é possível a obtenção de efeitos com longo alcance na capacidade do paciente de lidar com a doença, bem como na relação médicopaciente, afetando a adesão e possivelmente a eficácia futura das intervenções médicas (CUNHA VF e SCORSOLINI-COMIN F, 2019).

Em muitas pesquisas, em que foi investigado se os pacientes gostariam que os médicos perguntassem sobre suas crenças e necessidades espirituais, a resposta foi positiva. Em relação a posição dos médicos sobre tal aspectos, muitos acreditam que isso deveria fazer parte da anamnese do paciente, porém muitos não se sentem preparados e, portanto, não sabem como abordar tais aspectos durante uma consulta. As escolas médicas também apresentam essa característica. No currículo médico pouca atenção é dada as reflexões sobre as questões existenciais e os aspectos não biológicos do ser humano, sendo assim os estudantes são alertados quanto à necessidade de evitar a desumanização, mas não são instrumentalizados para tal (RIBEIRO LS, et al., 2021).

Ademais, no que tange a grade curricular das escolas médicas no Brasil, poucos possuem esses aspectos inclusos durante a formação acadêmica. Isso pode ser pela falta de conhecimento sobre o conteúdo, o que gera uma barreira por não encontrar embasamento e assim se sentirem despreparados para abordar tais temáticas em sua prática clínica ou mesmo por achar que tais assuntos devem ser tratados fora dos ambientes educacionais (CONDE SRSS, et al., 2019). Tal fator impossibilita o diálogo e a relação médico-paciente uma 
vez que a os profissionais direcionam o foco de seu trabalho apenas para a doença e não no sujeito e em suas dimensões, gerando assim desconforto, sentimento de desamparo e angústia nos pacientes (DOMINGUES MES, et al., 2020).

Esses aspectos também vêm sendo discutidos na Associação Mundial de Psiquiatria (WPA), que defendem a abordagem da espiritualidade e religiosidade dentro da história psiquiátrica do paciente, uma vez que isso se torna uma forma de enfrentamento e de compreensão do sentido existencial de cada ser, o que gera uma prática clínica baseada no cuidado humano e global (SCORSOLINI-COMIN F, 2018).

Outro fator a ser considerado é influência da relação dos profissionais na interação com o paciente e com os demais colegas, isso porque contribui para compreender os aspectos subjetivos do doente, promove um maior sentimento de harmonia e equilíbrio, fortalecendo os laços e impactando positivamente na assistência prestada (LONGUINIERE ACIFDL, et al., 2018).

Nessa perspectiva, considerando ainda que a formação do estudante de medicina é provavelmente um dos momentos mais importantes na sua carreira futura, o modo como a espiritualidade é retratada durante $o$ curso e a forma como tais estudantes analisam a sua importância irá exercer forte impacto na compreensão dessa dimensão e no uso desta na prática clínica. Por isso, é relevante trazer para o ensino não só a conscientização da necessidade de um estudo e da aplicação de tais temas, mas construir, discutir, publicar e aprender de forma crítica como isso pode ser aplicado durante a prática clínica (SCORSOLINI-COMIN F, 2018).

Assim, esse artigo teve como objetivo compreender as concepções dos estudantes de medicina sobre a influência da espiritualidade e religiosidade na prática clínica e o impacto destas na construção do cuidado e da relação médico-paciente.

\section{REVISÃO BIBLIOGRÁFICA}

A dimensão do cuidado na área da saúde, é usado para descrever as ações diretas ou indiretas de profissionais direcionadas a recuperação, preservação ou reabilitação da saúde de outrem. Diante disso, a formação acadêmica do profissional médico deve ser pautada primordialmente nesse cuidado integral e global do ser humano, contemplando, portanto, sua dimensão espiritual e religiosa, uma vez que a partir dessas é possível entender melhor o papel desse aspecto na vida do paciente, para prestar cuidados compassivos considerando a interação de fatores biopsicossociais na vida e na história espiritual de cada indivíduo (SILVA $A A$, et al., 2020).

Espiritualidade e religiosidade muitas vezes são empregadas como sinônimos em diversos trabalhos, no entanto, espiritualidade se baseia na busca individual por significado e sentido para a vida e de suas relações com o sagrado, pode ser considerada como uma filosofia da existência humana, gerando atitudes e sentimentos de compaixão, esperança e amor (SILVA LG, et al., 2020). Religiosidade, no entanto, se refere ao envolvimento do ser humano com um sistema organizado de crenças e práticas para se relacionar com 0 transcendente, engajando-o em uma filiação religiosa (CURCIO CSS e MOREIRA-ALMEIDA A, 2019).

Diante disso, o envolvimento nessas práticas impacta no psicológico dos pacientes, ao trazerem uma maior esperança, afetividade e paciência para lidar com o processo de busca da cura, sendo uma forma de enfrentar as adversidades, fortalecendo e confortando tanto os doentes quanto os familiares. Essa influência positiva é notada também fisiologicamente, na função cardíaca, endócrina, neuronal e imunológica, ao melhorar o eixo hormonal do estresse, o metabolismo dos lipídeos. Além disso, os pensamentos otimistas, positivos e de fortaleza vindos da confiança e das crenças proporcionam respostas fisiológicas positivas, como menores quantidades de Interleucina 6 (IL-6) e outros agentes inflamatórios, que medeiam reações de estresse, contribuindo assim para o processo de recuperação dos pacientes (SILVA LG, et al., 2020).

Além de fisiologicamente, a espiritualidade pode aflorar sentimentos de empatia, harmonia e com isso melhorar a relação tanto de estudantes em seu processo de construção de aprendizagem, quanto dos profissionais já em exercício, ao possibilitar uma melhor relação no trabalho e principalmente um maior conhecimento do paciente para o planejamento do cuidado em saúde (OLIVEIRA RM, et al., 2018). 
A abordagem médico-científico por si só gera algumas lacunas no conhecimento, uma vez que não oferece todas as explicações para as patologias e seus tratamentos, além de ser unicamente centrado no aspecto físico do adoecer, realizando um trabalho mecânico e singular do corpo, fragmentando a atenção ao paciente (FERREIRA AGC, et al., 2015). Com isso a parte espiritual e religiosa surge como um meio de suprir essa lacuna, pois facilita a compreensão de fatores até então inexplorados, além de trabalhar e facilitar a aceitação dos pacientes frente os desafios no processo de saúde-doença (SOUSA RS e AGUIAR MCM, 2021).

A aplicação desses conceitos e aspectos no âmbito do cuidado à saúde do indivíduo ultrapassa a visão mecanicista do homem, pautada muitas vezes em uma assistência fragmentada e limitada a aspectos científicos e puramente clínicos, e incorpora dimensões subjetivas, uma vez que o processo de saúde-doença é uma construção multidimensional, que inclui aspectos físicos, biológicos, sociais, psicológicos e espirituais (CARVALHO MS, et al., 2021).

No entanto, são escassas as pesquisas e os trabalhos publicados a respeito da construção de um modelo de saúde que permeie a espiritualidade e religiosidade na prática clínica e como essas questões influenciam no exercício de sua profissão. Tal fato dificulta a preparação dos estudantes sobre esse tema, visto que eles precisam entender o conceito espiritual e religioso como algo distante de julgamentos, concepções préestabelecidas e principalmente de preconceitos, uma vez que isso representa algo extremamente particularizado e individual (BORGES DC, et al., 2013).

Diante disso, os profissionais ligados à saúde precisam estar capacitados para atender os pacientes. Além disso, a espiritualidade pode levar a experiências no processo saúde-doença, se trabalhada nos contextos adequados. Por isso, os acadêmicos de medicina podem ver os benefícios na prática, quando compreenderem a importância disso para o cuidado e a atenção humanizada (COSTA MS, et al., 2019).

No que tange esse preparo, um estudo com estudantes de medicina demonstrou que a maioria dos entrevistados reconhecem a importância da temática na prática clínica e gostariam de realizar essa abordagem, embora a maior parte se sinta despreparada para fazê-lo (LUCCHETTI G, et al., 2013). Esse despreparo, representa a escassez de abordagem dessa temática durante o processo de formação profissional, isso porque existe uma lacuna no aprendizado e em pesquisa sobre o assunto (COSTA MS, et al., 2019).

Como consequência disso, cria-se um "círculo vicioso" entre falta de treinamento e insegurança de inserir a temática na prática clínica (RADDATZ JS, et al., 2019). Ademais, muitos coordenadores e professores do curso de medicina apontam que não sabem como introduzir esse treinamento. Algumas barreiras incluem um conhecimento superficial do assunto, falta de tempo, medo de fazer proselitismo ou de uma abordagem não científica e o desafio de adicionar mais um tópico a um currículo muito completo (OLIVEIRA FHAO, et al., 2021).

Essa deficiência na abordagem da religiosidade e espiritualidade durante a graduação vai ao oposto do panorama social e cultural do Brasil, uma vez que tais aspectos são muito debatidos e vividos pela população. Perde-se, portanto, uma oportunidade de debater com os estudantes tais aspectos para que futuramente possam aplicar e contribuir para um atendimento mais centrado no sujeito e nas suas individualidades (CARVALHO MS, et al., 2021).

Outra barreira encontrada é o receio de influenciar nas crenças do paciente e ser interpretado negativamente ao abordar tais temáticas na consulta, haja visto a diversidade de opiniões e manifestações religiosas existentes no meio social, exigindo, portanto, muito cuidado e atenção na forma de expressar e de agir do profissional (CARVALHO MS, et al., 2021). Além disso, estudantes apontam como barreiras adicionais, o medo de ofender os pacientes, desconforto e quebra do vínculo, falta de tempo e falta de relevância para o tratamento (SOUZA MAAF, et al., 2021).

Outro ponto considerado por muitos estudantes ou profissionais diz respeito ao momento e na fase em que o paciente se encontra e ainda a maneira como ele encara e vivencia a religiosidade e espiritualidade, exemplo disso, são alguns estados de psicose ou alucinação, onde debater sobre esses aspectos pode prejudicar ou até mesmo agravar o processo de tratamento (RODRIGUES DD, et al., 2020). 
Por tal fato, ao abordar esses aspectos, os profissionais e estudantes devem tomar cuidado com suas opiniões, culturas, manifestos religiosos e formas de significar as coisas, para evitar relações conflituosas e debates tendenciosos ou fantasiosas sobre o tema, o que provocaria tensão e desconforto para os pacientes. Ou seja, para que seja abordado esse tema nos atendimentos, o profissional deve deixar de lado suas próprias crenças e filiações religiosas, para compreender e considerar a opinião de seu paciente, uma vez que a imposição e a influência particular de suas práticas religiosas podem oferecer um risco e uma quebra do processo de ética médica (CUNHA VF e SCORSOLINI-COMIN F, 2019).

Assim, existe alternativas para que o tema espiritualidade e religiosidade possa ser debatido e estudado durante a graduação, como: oferecer na matriz curricular assuntos que discutam a importância e essência do tema na prática profissional, oferecer cursos e estágios complementares sobre o tema e a realização de projetos como debates, seminários onde se discuta a importância do tema na prática clínica, bem como a fundação de ligas acadêmicas específicas para estudo e pesquisa na área (SOUSA RS e AGUIAR MCM, 2021).

Com isso, é de extrema importância que o educador dê a oportunidade para que os estudantes revelem suas opiniões e posições sobre esses conteúdos, estimulando que eles tomem consciência e exteriorizem seus sentimentos, vivencias e sua perspectiva, demonstrando assim o potencial que cada um tem de fornecer esse cuidado que vai além do saber técnico (HARMUCH C, et al., 2019).

Nessa perspectiva, considerando ainda que a formação do estudante de medicina é provavelmente um dos momentos mais importantes na sua carreira futura, o modo como a espiritualidade é retratada durante 0 curso e a forma como tais estudantes analisam a sua importância irá exercer forte impacto na compreensão dessa dimensão e no uso desta na prática clínica. Essa proposta demonstra o envolvimento do profissional com as questões espirituais e religiosas desde sua formação, compreendendo como relevantes não apenas as questões do paciente, mas as do próprio profissional de saúde, uma vez que a partir dele deve partir o respeito, atenção e compreensão de tais aspectos (TROFA GC, et al., 2021).

Além disso, abordar esses aspectos durante a graduação, proporciona o contato dos acadêmicos com essa temática, para que eles se apropriem do assunto, desenvolvam seus valores e opiniões e consigam ter maturidade e discernimento para abordar esse tema em sua posterior prática clínica (OLIVEIRA RM, et al., 2018).

Dessa forma, ainda que abordar o paciente do ponto de vista global, abarcando os diversos aspectos de sua individualidade e relação com o meio, fortalece a relação médico-paciente e permite a criação de leques terapêuticos, diagnósticos e preventivos. Assim, uma vez que o processo de tratamento se torna mais fácil uma vez que eles possuem uma rede de apoio, amigos e familiares que estejam em consonância com o doente, crenças, valores, práticas e experiências religiosas e meios que proporcionam um maior significado para a vida desse paciente e o motive durante seu processo de busca terapêutica (GOMES SB, et al., 2018).

É importante destacar que abordar espiritualidade e religiosidade é tornar a prática médica mais humanizada, uma vez que mesmo quando a ciência e os tratamentos não conseguem resolver o problema do paciente, o simples fato de ouvir, dar apoio e garantir que essa pessoa vivencie sua espiritualidade pode Ihe proporcionar conforto e consolo e assim contribuir para a construção de uma medicina baseada no cuidado (REGINATO V, et al., 2016). Além disso, muitas vezes a espiritualidade e religiosidade é um fator intrínseco à grande parcela da população e quando o profissional aprende a trabalhar com essas questões, ele poderá potencializar seu atendimento e facilitar a construção do vínculo entre médico e paciente (FERREIRA TT, et al., 2018).

Entretanto, mesmo diante dos pontos positivos demostrados pela religiosidade e espiritualidade na prática clínica, os profissionais precisam tomar alguns cuidados em certos casos, uma vez que alguns pacientes podem se negar a usar o tratamento formal prescrito e colocar como ápice suas crenças e saberes próprios, isso ofereceria riscos à recuperação e na própria relação entre médico-paciente (CUNHA VF e SCORSOLINICOMIN F, 2019, CUNHA VF, et al., 2021).

Associado a isso, é necessário o enfrentamento das questões de saúde de forma ampla, visto que ela representa um campo interdisciplinar, que necessita de conhecimento de múltiplas áreas. Pois isso 
proporciona um maior resgate na relação médico-paciente (KOLODIY T e PONTíFICE OS, 2019). Ademais, atividades e crenças religiosas estão relacionadas à melhor saúde e qualidade de vida e podem ser responsáveis pela maior aderência aos medicamentos, melhoria nos hábitos de vida, relacionados a alimentação e prática de exercícios físicos, impactando portando no processo terapêutico (THIENGO PCS, et al., 2019).

Ainda assim, existe uma grande dificuldade em saber o momento certo de abordar a espiritualidade de um paciente e assim poupar um mal-entendido, o bom senso deve imperar. Isso porque, em situações mais extremas, esses questionamentos podem proporcionar medo e desespero, enquanto em outras pode ocasionar esperança e confiança. Durante a anamnese, esse questionamento sobre religiosidade e espiritualidade pode acompanhar a história social e hábitos de vida do paciente. Assim, não existe uma só forma de abordar a espiritualidade e não se tem uma forma correta, pois deve ser feita de uma forma natural e tranquila durante a consulta, porém isso irá sofrer influência dos aspectos culturais tanto do médico quanto do paciente (SOUZA MCA, et al., 2019).

Em pacientes não praticantes de nenhuma religião, por exemplo, ao invés de questionar diretamente sobre esse aspecto, o profissional pode indagar sobre como essa pessoa convive com a doença, ou o que pra ele significa sua existência, quais são seus prazeres, atividades de lazer, meios culturais, fatores que podem influenciar positivamente no processo terapêutico. Outra associação positiva entre espiritualidade/ religiosidade e saúde foi na formulação de bons hábitos (SOUSA RS e AGUIAR MCM, 2021).

Estudos demonstram uma relação direta entre espiritualidade, melhores índices de atividade física, alimentação saudável, redução do tabagismo e consumo de drogas. Existe uma forte associação entre religiosidade e melhor saúde mental, principalmente em situações de alto estresse, sendo constatado, portanto, menor incidência de ansiedade e depressão nesse grupo de pacientes (CUNHA VF, et al., 2021; MENDONÇA SDG, 2021, GOMES SB, et al., 2018).

Sendo assim, os pacientes querem ser vistos e tratados como pessoas inteiras, não como doenças, tendo, portanto, dimensões físicas, emocionais e espirituais. Ignorar alguma dessas partes, faz com que o indivíduo se sinta incompleto o que pode impactar negativamente o processo de tratamento. Os profissionais da saúde, devem se atentar, respeitar e saber abordar as questões religiosas e espirituais de seus pacientes, para que a prática do cuidado seja efetiva, holística e totalmente humanizada (GOMES ET e BEZERRA SMMS, 2020).

Dessa forma, percebe-se que o processo de cura muitas vezes vai além de tratamentos técnicos e propriamente físicos, sendo necessário usar outros leques terapêuticos que perpassam sobre a mente e sobre os aspectos humanos do sujeito. A espiritualidade e a religiosidade entram, portanto, como ferramenta para enfrentar esses momentos. Por isso, estudantes e profissionais médicos devem estar aptos e abertos para encaixar tais aspectos dentro da rotina de atendimentos, visto que é essencial que essa discussão se abra e assim proporcione o aumento no vínculo entre médico-paciente e facilite a adesão ao tratamento (OLIVEIRA GR, et al., 2013).

\section{CONSIDERAÇÕES FINAIS}

Ao considerar o ser humano em sua totalidade, abarcando suas individualidades, crenças e suas atitudes com o meio, é possível ampliar e enriquecer a relação com o paciente e, portanto, facilitar a tomada de decisões durante a prática clínica. Essa realidade denota que a religiosidade e espiritualidade, quando bem interpretadas e utilizadas, podem fornecer informações essenciais para compreensão de questões atuais do paciente e ainda impactar positivamente em todas as etapas do processo de cuidado. Além disso, é necessário que essa percepção seja validade e construída durante a formação do estudante, garantindo que os posteriores profissionais médicos entendam melhor o papel desses aspectos na assistência ao paciente.

\section{REFERÊNCIAS}

1. BORGES DC, et al. Saúde, espiritualidade e religiosidade na visão dos estudantes de medicina. Revista Brasileira de Clínica Médica, 2013; 11(1): 6-11.

2. CARVALHO MS, et al. A importância da fé para auxiliar a cura na medicina: relato de caso introdução. Brazilian Journal of Health Review, 2021; 4(3): 6964-9668. 
3. CONDE SRSS, et al. A espiritualidade nos currículos das escolas médicas da região norte e a visão do interno de medicina sobre sua importância na formação. Interdisciplinary Journal of Health Education, 2019; 4(1-2): 9-18.

4. COSTA MS, et al. Espiritualidade e religiosidade: saberes de estudantes de medicina. Revista Bioética, 2019; 27(2): 350-358.

5. CUNHA VF, et al. Religião, religiosidade, espiritualidade, ancestralidade: tensões e potencialidades no campo da saúde. Revista Relegens Thréskeia, 2021, 10(1): 143-170.

6. CUNHA VF, SCORSOLINI-COMIN F. A dimensão religiosidade/espiritualidade na Prática Clínica: revisão Integrativa da literatura científica. Psicologia: Teoria e Pesquisa, 2019; 35: 1-12.

7. CURCIO CSS, MOREIRA-ALMEIDA A. Investigação dos conceitos de religiosidade e espiritualidade em amostra clínica e não clínica em contexto brasileiro: uma análise qualitativa. Interação em Psicologia, 2019; $23(2): 281$-292.

8. DOMINGUES MES, et al. Religião, religiosidade e espiritualidade e sua relação com a saúde mental em contexto de adoecimento: uma revisão integrativa de 2010 a 2020. Caderno Programa de Alfabetização na Idade Certa, 2020; 21(1): 573-594.

9. FERREIRA AGC, et al. Concepções de espiritualidade e religiosidade e a prática multiprofissional em cuidados paliativos. Revista Kairós: Gerontologia, 2015; 18(3): 227-244.

10. FERREIRA TT, et al. Percepção de acadêmicos de medicina e de outras áreas da saúde e humanas (ligadas à saúde) sobre as relações entre espiritualidade, religiosidade e saúde. Revista Brasileira de Educação Médica, 2018; 42(1): 67-74.

11. GOMES ET, BEZERRA SMMS. Espiritualidade, integralidade, humanização e transformação de paradigma do campo da saúde no Brasil. Revista Enfermagem Digital Cuidado e Promoção da Saúde, 2020; 5(1): 65-69.

12. GOMES SB, et al. Avaliação da influência da espiritualidade e religiosidade no processo saúde doença. Saúde em foco, 2018; 3: 115-128.

13. HARMUCH C, et al. Religião e espiritualidade no ensino e assistência de enfermagem na visão dos estudantes: uma revisão. Revista Uningá, 2019; 56(2): 243-254.

14. KOLODIY T, PONTÍFICE PS. A dimensão espiritual nos cuidados de saúde à pessoa em situação crítica e sua família: revisão integrativa. Servir, 2019; 60(1-2): 47-58.

15. LONGUINIERE ACIFDL, et al. Influência da religiosidade/espiritualidade do profissional de saúde no cuidado ao paciente crítico. Revista Cuidarte, 2018; 9(1): 1961-1972.

16. LUCCHETTI G, et al. Medical students, spirituality and religiosity-results from the multicenter study SBRAME. BMC Medical Education, 2013; 13: 1-8.

17. MENDONÇA SDG. A relevância da espiritualidade em transtornos depressivos. Revista Científica Rumos da inFormação, 2021; 2(1): 46-62.

18. NANTES AC, GRUBITS S. A religiosidade/espiritualidade como um possível fator de ajuda à prevenção da prática suicida. Revista Contemplação, 2017; (16): 73-84.

19. OLIVEIRA FHAO, et al. Religiosidade e espiritualidade em programas de residência em psiquiatria: por que, o que e como ensinar?. Brazilian Journal of Psychiatry, 2021, 43(4): 424-430.

20. OLIVEIRA GR, et al. Saúde, espiritualidade e ética: a percepção dos pacientes e a integralidade do cuidado. Buscando a excelência na disseminação do conhecimento científico, 2013; 11(2): 140-144.

21. OLIVEIRA RM, et al. Espiritualidade/religiosidade e o humaniza SUS em Unidades de Saúde da Família. Revista Brasileira em Promoção da Saúde, 2018; 31(1): 1-8.

22. RADDATZ JS, et al. Religiosidade/Espiritualidade na Prática Clínica: Círculo Vicioso entre Demanda e Ausência de Treinamento. Psicologia - Universidade São Francisco, 2019; 24(4): 699-709.

23. REGINATO V, et al. Espiritualidade e saúde: uma experiência na graduação em medicina e enfermagem. Trabalho, Educação e Saúde, 2016; 14(1): 237-255.

24. RIBEIRO LS, et al. Espiritualidade no ensino: a perspectiva dos discentes de uma universidade pública da área da saúde. Educação, 2021; 46: 3-24.

25. RODRIGUES DD, et al. Religiosidade e espiritualidade na prática clínica em saúde mental. Revista Eletrônica Acervo Saúde, 2020; 12(7): e3327-e3327.

26. SCORSOLINI-COMIN F. A religiosidade/espiritualidade no campo da saúde. Health Sciences Journal, 2018; 8(2): 12.

27. SILVA AA, et al. A espiritualidade no processo de formação dos profissionais de saúde. Revista Pró-UniverSUS, 2020, 11(2): 158-163.

28. SILVA LG, et al. Relação entre medicina e espiritualidade/religiosidade: impacto no processo de adoecimento. Revista Uningá, 2020; 57(4): 93-100.

29. SOUSA RS, AGUIAR MCM. A influência do curso de medicina na espiritualidade dos estudantes. Revista PróUniverSUS, 2021; 12(2): 78-85.

30. SOUZA MAAF, et al. Percepção de acadêmicos de medicina sobre a relação entre espiritualidade, religiosidade e saúde. Brazilian Journal of Development, 2021; 7(12): 111390-111405.

31. SOUZA MCA, et al. A espiritualidade no cuidado em saúde na Atenção Primária. Revista Pró-UniverSUS, 2019; 10(2): 70-74.

32. THIENGO PCS, et al. Espiritualidade e religiosidade no cuidado em saúde: revisão integrativa. Cogitare Enfermagem, 2019; 24: e58692.

33. TROFA GC, et al. A espiritualidade/religiosidade como desafio ao cuidado integral: aspectos regulatórios na formação médica brasileira. Physis: Revista de Saúde Coletiva, 2021; 31(4): 555-576. 